\title{
APLICAÇÃO DE HERBICIDA EM DIFERENTES VOLUMES E HORÁRIOS NO CONTROLE DE PLANTAS DANINHAS
}

\author{
Bruno Mallmann', Jackeline Matos Nascimento', Paulo Monteiro Boeira Barbosa ${ }^{1}$, Adriana \\ Vianna Shwann Stoffel ${ }^{1}$, Sálvio Napoleão Soares Arcoverde ${ }^{1}$
}

${ }^{1}$ Centro Universitário da Grande Dourados - UNIGRAN, Dourados, 79824-900, Mato Grosso do Sul, Brasil

Autor para correspondência: Jackeline Matos Nascimento; e-mail: jackeline_ms@yahoo.com.br

Recebido: 20/05/2020, Aceito: 0606/2021

\begin{abstract}
Resumo
O controle de plantas daninhas é uma prática muito utilizada no sistema de semeadura direta de cereais, sendo o uso de herbicidas indispensável para uma correta deposição de produto na parcela. O objetivo deste trabalho foi avaliar diferentes volumes e horários de aplicações de herbicida no controle de plantas daninhas. O experimento foi instalado em esquema de parcelas subdivididas, com três volumes de aplicação $\left(160,130\right.$ e $\left.100 \mathrm{~L} \mathrm{ha}^{-1}\right)$ e 2 horários (7 e 10 horas). $\mathrm{Na}$ aplicação foram utilizados: 2,4 diclorofenoxiacético, metsulfuron-methyl e glifosato potássico nas doses recomendadas pelo fabricante, além do adjuvante lauril éter sulfato de sódio $100 \mathrm{~mL} \mathrm{ha}^{-1}$. Analisaram-se dados de tecnologia de aplicação e controle de Cenchrus echinatus L., Bidens pilosa L. e Digitaria horizontalis Willd, aos 7, 14 e 21 dias após aplicação. Quando foi realizada a aplicação com volume de $160 \mathrm{~L} \mathrm{ha-}$ ${ }^{1}$ verificaram-se os melhores resultados para o diâmetro da mediana volumétrica, o volume recuperado, a porcentagem de área coberta e a densidade de gotas. Para estes parâmetros, houve efeito dos horários quando aplicado o volume de $100 \mathrm{~L} \mathrm{ha}^{-1}$. A aplicação realizada no horário das 10 horas proporcionou melhor eficiência no controle das espécies de plantas daninhas avaliadas.
\end{abstract}

Palavras-chave: Cenchrus echinatus L., Bidens pilosa L., Digitaria horizontalis Willd

\section{APPLICATION OF HERBICIDES IN DIFFERENT VOLUMES AND PERIODS IN WEED CONTROL}

\begin{abstract}
Weed control using herbicides is a highly common practice in direct seeding systems on cereal grains crops. The study of the application technology of these types of herbicides has become essential when it comes to calculating the application rates of herbicide. The objective of this work was to evaluate the application rate of herbicide in different periods of the day. The experiment was designed in two threads with three application rates $(160,130$, and $1001 \mathrm{ha}^{-1}$ ), at two different times (7:00 am and 10:00 am). It was utilized in the application 2,4Dichlorophenoxyacetic acid, Metsulfuron-methyl, and Glyphosate at the recommended levels of usage. It was also used sodium laureth sulfate $\left(100 \mathrm{ml} \mathrm{ha}^{-1}\right)$. Technology application and control data were analysed on the following weeds submitted to herbicide application: Cenchrus echinatus L., Bidens pilosa L. e Digitaria horizontalis Willd, at 7, 14, and 21 days after application. To parameters such as volume mean diameter, volume recovery, covered area, and droplet density, the best results were associated with the application rate of $1601 \mathrm{ha}^{-1}$. When different periods of application were considered, there were only distinct effects for the application rate of $100 \mathrm{l} \mathrm{ha}^{-1}$. In order to control weed, applications of herbicide at 10:00 am showed to be more effective for the types of weeds evaluated.
\end{abstract}

Keywords: Cenchrus echinatus L., Bidens pilosa L., Digitaria horizontalis Willd. 


\section{Introdução}

As plantas daninhas, espécies vegetais que se desenvolvem onde não são desejadas, representam um dos fatores limitantes ao potencial produtivo das culturas. O prejuízo do potencial produtivo decorre do processo de interferência, da competição que ocorre quando água, luz e nutrientes se tornam limitantes ao crescimento das plantas, resultando em prejuízos (CHRISTOFFOLETI, 2004). O controle dessas plantas daninhas consiste na adoção de práticas que resultam na redução da infestação. Esta redução da interferência das plantas daninhas em uma cultura deve ser feita até o nível em que as perdas pela interferência sejam iguais ao custo do controle, ou seja, de modo que não influenciem a produção econômica da cultura (SILVA et al. 1999). Apesar do controle químico de plantas daninhas ser considerado o método mais eficiente, quando não se utiliza corretamente a tecnologia de aplicação, esta eficiência é comprometida. Uma aplicação mal feita pode aumentar o banco de sementes das espécies não controladas, além da perda com o produto comercial bem como os gastos para realizar a aplicação, como também da contaminação ambiental (NIEWEGLOWSKI FILHO, 2014).

Para melhor aplicação de herbicidas torna-se necessário o conhecimento da tecnologia de aplicação. As aplicações devem colocar o produto biológico ativo (ou ingrediente ativo) direto no alvo de forma eficiente (CUNHA, 2008). Estima-se que cerca de $50 \%$ dos defensivos são desperdiçados devido às más condições de aplicação (FRIEDRICH, 2004). A tecnologia consiste na aplicação dos conhecimentos científicos a um determinado processo produtivo, logo, entende-se como Tecnologia de Aplicação de Produtos Fitossanitários o emprego de todos os conhecimentos científicos que proporcionam a correta colocação do produto biologicamente ativo no alvo, em quantidade necessária, de forma econômica, com o mínimo de contaminação de outras áreas (MATUO, 1990).

A não observação destas técnicas resulta em dosagens dos herbicidas muitas vezes diferentes da recomendação, para que o controle de plantas daninhas seja efetivamente condições ambientais e de manejo e, em especial, devido às falhas na tecnologia de aplicação (DEVLIN, 1991). Usando-se técnicas e métodos de aplicação adequados, limpeza e regulagem do equipamento, aplicação em condições meteorológicas adequadas e/ou uso de formulação apropriada pode-se minimizar significativamente a deriva (FOLONI, 2000).

Atualmente, há uma tendência de redução dos volumes de calda, a fim de diminuir os custos de produção e elevar a capacidade operacional das aplicações, aumentando a autonomia e dos pulverizadores e diminui o risco de perdas por escorrimento (RODRIGUES, 2011). Contudo, essa redução pode levar a uma menor cobertura do alvo, comprometendo a eficácia dos tratamentos. Embora seja prática comum em algumas propriedades, a redução do volume de calda ainda desperta incerteza sobre sua viabilidade e limite mínimo. Dessa forma, é essencial conhecer as características físico-químicas das caldas de pulverização de forma a entender a função de seus componentes, uma vez que existe pouca informação a esse respeito e sobre os fatores que as influenciam (SCHAMPHELEIRE et al., 2009).

A absorção foliar é um fator determinante na ação dos herbicidas aplicados em pós-emergência, podendo também ser influenciada por vários fatores ambientais, como temperatura, luminosidade, umidade relativa do ar, ventos, presença de orvalho e de chuva, os quais determinarão uma maior ou menor permeabilidade da cutícula das plantas e, em consequência disto, na quantidade do produto absorvido (RAMIRES et al., 1999). De forma geral, os períodos do início da manhã, fim da tarde e à noite são os horários mais adequados para a aplicação de defensivos, pois a temperatura é menor e a umidade relativa maior, mas $\mathrm{o}$ monitoramento das condições ambientais é indispensável (ANTUNIASSI, 2015).

O monitoramento das condições meteorológicas é indispensável antes da aplicação, entretanto durante o dia estas condições podem ser alteradas sendo que o horário da manhã é considerado o mais efetivo para a pulverização, resultando na eficácia da aplicação e ganho de produtividade (FAVARETTO, 2019). As condições climáticas adequadas são velocidade vento sempre menor que $8 \mathrm{~km} \mathrm{~h}^{-1}$, umidade maior que $55 \%$, a temperatura menor que $30^{\circ} \mathrm{C}$, (ADEGAS, 2016).

Neste contexto, o objetivo deste trabalho foi avaliar diferentes volumes e horários de aplicações de herbicida no controle de plantas daninhas, antecedendo a semeadura da aveia.

\section{Material e Métodos}

O experimento foi conduzido na fazenda Barigui em Amambaí-MS, com as coordenadas, latitude $23^{\circ} 11^{`} \mathrm{~S}$, longitude de $55^{\circ} 19^{\circ} \mathrm{W}$ e altitude de $480 \mathrm{~m}$. O solo da área comercial é descrito como Latossolo Vermelho Escuro distrófico e textura média. As aplicações foram realizadas no dia 17 de abril de 2017, quando as plantas daninhas se encontravam com aproximadamente 20 dias após emergência (LORENZI, 2014). As espécies de maior ocorrência na área foram capim-carrapicho (Cenchrus echinatus L.), picão preto (Bidens pilosa L.) e capim-colchão (Digitaria horizontalis Willd.).

Os herbicidas utilizados foram o 2,4 diclorofenoxiacético, metsulfuron-methyl e glifosato 
potássico, nas doses recomendadas pelo fabricante $(0,8$ $\mathrm{L} \mathrm{ha}^{-1}, 4 \mathrm{~g} \mathrm{ha}^{-1}$ e 3,5 $\mathrm{L} \mathrm{ha}^{-1}$ respectivamente), além do adjuvante lauril éter sulfato de sódio $100 \mathrm{~mL} \mathrm{ha}^{-1}$.

O delineamento experimental utilizado foi o de blocos casualizados e os tratamentos foram dispostos em esquema de parcelas subdivididas, onde as parcelas foram volumes (100, 130 e $\left.150 \mathrm{~L} \mathrm{ha}^{-1}\right)$ e as subparcelas os horários de aplicação (07:00 e 10:00h) sendo as 7:00 na maioria dos dias um horário em que iniciam-se as aplicações devido as condições meteorológicas serem adequadas a aplicação e as 10:00, horário em que, dependendo do tamanho da propriedade é finalizada a aplicação, com 6 repetições.

A aplicação foi realizada com um pulverizador autopropelido com velocidade de $8 \mathrm{~km} \mathrm{~h}^{-1}$, com uma barra de 21 metros e com 42 bicos (ponta duplo leque plano Magnojet MUG110 02, com 1,2 $\mathrm{L} \mathrm{min}^{-1}$ de vazão) espaçados em 0,5 metros, altura da barra de 0,8 metros. Para avaliação de plantas daninhas, houve um tratamento adicional (testemunha sem aplicação do herbicida). A parcela experimental possuía 30 metros de comprimento e 10,5 metros de largura (meia barra).

As condições climáticas foram monitoradas com auxílio de termo-higrômetro digital (Tabela 1). A velocidade do vento durante as aplicações foi monitorada com auxílio de Anemômetro de Molinete.

Tabela 1: Condições climáticas observadas no horário da instalação do experimento, temperatura e umidade relativa do ar (Amambai-MS).

\begin{tabular}{cccc}
\hline Horário & $\mathrm{T}\left({ }^{\circ} \mathrm{C}\right)$ & $\mathrm{UR}(\%)$ & Velocidade do vento $\left(\mathrm{Km} \mathrm{h}^{-1}\right)$ \\
\hline $7 \mathrm{~h}$ & 20,5 & 82 & 6,0 \\
$10 \mathrm{~h}$ & 22,8 & 85 & 6,0 \\
\hline
\end{tabular}

Para a avaliação de tecnologia de aplicação foram utilizados papeis sensíveis a água e óleo (TeeJet, dimensões $26 \times 76 \mathrm{~mm}$ ), fixados horizontalmente com grampeador sobre a planta localizada na área útil da parcela. Após as aplicações, os papeis foram armazenados em caixa com sílica-gel, para evitar que a umidade do ambiente interferisse nos resultados. Estes foram digitalizados com resolução de $600 \mathrm{dpi}$, posteriormente sendo avaliados no programa Gotas (EMBRAPA, 2006) por meio do qual determinou-se os parâmetros: diâmetro da mediana volumétrica (DMV), porcentagem de cobertura (\%) e densidade de gotas (número de gotas $\mathrm{cm}^{-2}$ ).

As avaliações de controle de plantas daninhas foram efetuadas visualmente aos 7, 14 e 21 dias após a aplicação dos tratamentos. A metodologia de avaliação utilizada foi visual, no qual comparou-se as parcelas que receberam os tratamentos com a parcela testemunha, em que $0 \%$ correspondeu a plantas sem sintomas de fitotoxidez e $100 \%$ a plantas mortas (VELINI, OSIPE e GRAZZIERO, 1995).

Os resultados foram submetidos a análise de variância e as médias comparadas pelo teste Tukey $(P \leq$ $0,05)$.

\section{Resultados e Discussão}

No momento da instalação do experimento e nos dois horários de aplicação havia condições climáticas favoráveis à aplicação, em que as temperaturas médias e umidade relativa foram, respectivamente, de $20,5^{\circ} \mathrm{C}$ e $82 \%$ para o horário das 7 horas e de $22,8^{\circ} \mathrm{C}$ e $85 \%$ no horário das 10 horas. Os horários recomendados para uma aplicação eficiente de acordo com Matthews et al. (2016) são aqueles nos quais as temperaturas são inferiores a $30{ }^{\circ} \mathrm{C}$, a umidade relativa do ar superior a $50 \%$ e os ventos constantes de 3,2 a $6,5 \mathrm{Km} \mathrm{h}^{-1}$.

Na Tabela 2 estão apresentados os dados referentes ao diâmetro da mediana volumétrica (DMV), que representa o diâmetro da gota tal que metade do volume pulverizado é constituída de gotas de maior diâmetro e a outra metade, gotas de menor diâmetro (SANTOS, 2005).

$\mathrm{Na}$ avaliação do diâmetro de gotas os papeis que receberam $160 \mathrm{~L} \mathrm{ha}^{-1}$ de volume de calda, apresentaram maior DMV nos dois horários de aplicação. Apesar de todos os diâmetros de gotas $(\mu \mathrm{m})$ estarem dentro da classificação de gotas, como muito finas, ou seja, menores do que $119 \mu \mathrm{m}$ (ASABE, 2021), o volume 160 $\mathrm{L} \mathrm{ha}^{-1}$ embora tenha a mesma classificação de diâmetro de gotas que os demais volumes estudados, proporcionou gotas maiores e quanto maior a gota maior o tempo de vida e menor capacidade de deriva (SILVA et al., 2009). Não houve diferença para horário de aplicação. De acordo com Antuniassi e Baio (2008) o tamanho da gota é um dos parâmetros fundamentais para o sucesso na aplicação de defensivos, pois possui influência sobre a deposição e as perdas dos produtos por deriva.

Para o parâmetro volume recuperado e porcentagem de área coberta verificou-se significância somente para horários de aplicação, sendo maior para parcelas que receberam herbicidas às $10 \mathrm{~h}$. Nos dois horários as condições ambientais estavam favoráveis à aplicação, porém às $10 \mathrm{~h}$ a umidade do ar era maior quando comparadas às $7 \mathrm{~h}$, com isso, ocorreram menores perdas por evaporação das gotas menores, aumentando a densidade de gotas obtendo assim maior volume recuperado para horários com condições climáticas mais adequadas. Resultados semelhantes aos verificados por Nascimento (2009). Nascimento et al. (2013) observaram que pontas de pulverização que produzem gotas finas têm melhor porcentagem de 
cobertura e densidade de gotas quando a aplicação ocorre em condições climáticas favoráveis.

Tabela 2: Diâmetro da mediana volumétrica, volume de calda recuperado, porcentagem de área coberta e densidade de gotas (número de gotas $\mathrm{cm}^{-2}$ ) em papeis hidrossensíveis.

\begin{tabular}{|c|c|c|c|c|c|c|c|c|}
\hline \multirow[t]{2}{*}{ Volume (L ha $\left.{ }^{-1}\right)$} & \multicolumn{2}{|c|}{$\mathrm{DMV}(\mu \mathrm{m})$} & \multicolumn{2}{|c|}{$\begin{array}{l}\text { Volume } \\
\text { recuperado }\end{array}$} & \multicolumn{2}{|c|}{$\begin{array}{c}\% \text { de Área } \\
\text { Coberta }\end{array}$} & \multicolumn{2}{|c|}{$\mathrm{N}^{\circ}$ de Gotas $\mathrm{cm}^{-2}$} \\
\hline & $7 \mathrm{~h}$ & $10 \mathrm{~h}$ & $7 \mathrm{~h}$ & $10 \mathrm{~h}$ & $7 \mathrm{~h}$ & $10 \mathrm{~h}$ & $7 \mathrm{~h}$ & $10 \mathrm{~h}$ \\
\hline 160 & $118 \mathrm{a}$ & $115,6 \mathrm{a}$ & $212 \mathrm{~A}$ & $215 \mathrm{~A}$ & $31,5 \mathrm{~A}$ & $31,1 \mathrm{~A}$ & $99,5 \mathrm{aA}$ & $98,0 \mathrm{aA}$ \\
\hline 130 & $79,6 \mathrm{~b}$ & $95,1 \mathrm{ab}$ & $205 \mathrm{~A}$ & $236 \mathrm{~A}$ & $27,4 \mathrm{~A}$ & $30,1 \mathrm{~A}$ & $69,0 \mathrm{bA}$ & $98,0 \mathrm{abA}$ \\
\hline 100 & $70,1 \mathrm{~b}$ & $74,3 \mathrm{~b}$ & $189 \mathrm{~B}$ & $237 \mathrm{~A}$ & $26,0 \mathrm{~B}$ & $32,3 \mathrm{~A}$ & $51,3 \mathrm{bB}$ & $57,3 \mathrm{bA}$ \\
\hline CV horários (\%) & \multicolumn{2}{|c|}{15,48} & \multicolumn{2}{|c|}{15,7} & \multicolumn{2}{|c|}{5,99} & \multicolumn{2}{|c|}{29,9} \\
\hline CV volumes (\%) & \multicolumn{2}{|c|}{16,89} & \multicolumn{2}{|c|}{14,23} & \multicolumn{2}{|c|}{4,89} & \multicolumn{2}{|c|}{14,98} \\
\hline
\end{tabular}

Condições climáticas interferem na aplicação, agindo diretamente sobre as gotas, de maneira a alterar o deslocamento destas em direção ao alvo (QASEM, 2011). Entretanto, os efeitos das condições climáticas nas aplicações de herbicidas têm sido estudados principalmente quanto à eficácia e à absorção foliar, levando-se em consideração geralmente o horário das aplicações, como se pode constatar no trabalho de Penckowski, Podolan e Lópezovejero (2003).

Houve interação significativa entre horário de aplicação e volumes de calda para avaliação de porcentagem de área coberta, no qual a aplicação realizada no horário das 10 horas proporcionou maior volume recuperado corroborando com os resultados de Almeida et al. (2014), testando diferentes volumes de calda de glifosato no controle de Conyza sp., concluíram que com volumes de calda maiores proporcionaram uma melhor qualidade na aplicação, considerando parâmetros densidade de gotas e porcentagem de cobertura, em momentos que as condições atmosféricas foram desfavoráveis.

$\mathrm{Na}$ densidade de gotas, o volume de $160 \mathrm{~L} \mathrm{ha}^{-1}$ foi significativamente maior quando comparado ao volume de $100 \mathrm{~L} \mathrm{ha}^{-1}$ nos dois horários de aplicação. A menor densidade de gotas foi observada quando a aplicação foi realizada as $7 \mathrm{~h}$, com volume de $100 \mathrm{~L} \mathrm{ha}^{-1}$. Nos volumes de 130 e $160 \mathrm{~L} \mathrm{ha}^{-1}$ não houve diferença significativas. O número de gotas por unidade de área está intimamente relacionado com o volume aplicado, correlacionando-se positivamente (MATTHEWS et al., 2014).

O controle de Digitaria horizontalis avaliado está descrito na Tabela 3. Não houve significância para os volumes, o que infere que a redução no volume de calda não interfere negativamente na eficiência do controle químico. Para os horários de aplicação houve melhor eficácia quando os produtos foram aplicados as $10 \mathrm{~h}$ nas avaliações de 7 e 14 dias após a aplicação, aos 21 dias após a aplicação houve o controle de $100 \%$ do capim colchão. Para maiores volumes de aplicação a calda se torna menos concentrada, e assim proporciona gotas com menor concentração do ativo, podendo influenciar a eficiência de controle em relação aos volumes menores na aplicação de herbicidas com maior mobilidade em plantas, entretanto, independente do volume de calda a quantidade de ingrediente ativo por hectare que chega no alvo a ser controlado é o mesmo (ALMEIDA et al., 2014).

Tabela 3: Efeito do volume e horário de aplicação no controle da Digitaria horizontalis aos 7, 14 e 21 dias após aplicação de herbicida. Amambai-MS, 2017.

\begin{tabular}{|c|c|c|c|c|c|c|}
\hline \multirow{3}{*}{$\begin{array}{l}\text { Volume } \\
\text { (L ha-1) }\end{array}$} & \multicolumn{6}{|c|}{ Controle (\%) } \\
\hline & \multicolumn{2}{|c|}{7 dias } & \multicolumn{2}{|c|}{14 dias } & \multicolumn{2}{|c|}{21 dias } \\
\hline & $7 \mathrm{~h}$ & $10 \mathrm{~h}$ & $7 \mathrm{~h}$ & $10 \mathrm{~h}$ & $7 \mathrm{~h}$ & $10 \mathrm{~h}$ \\
\hline 160 & $71,6 \mathrm{~B}$ & $76,0 \mathrm{~A}$ & $97,0 \mathrm{~B}$ & $99,3 \mathrm{~A}$ & 100 & 100 \\
\hline 130 & $71,3 \mathrm{~B}$ & $76,3 \mathrm{~A}$ & 97,3 B & $99,0 \mathrm{~A}$ & 100 & 100 \\
\hline 100 & $71,0 \mathrm{~B}$ & $76,3 \mathrm{~A}$ & $97,3 \mathrm{~B}$ & $99,0 \mathrm{~A}$ & 100 & 100 \\
\hline $\begin{array}{c}\text { CV horários } \\
(\%)\end{array}$ & \multicolumn{2}{|c|}{0,64} & \multicolumn{2}{|c|}{0,51} & \multicolumn{2}{|c|}{0} \\
\hline $\begin{array}{c}\text { CV volumes } \\
(\%)\end{array}$ & \multicolumn{2}{|c|}{0,71} & \multicolumn{2}{|c|}{0,71} & \multicolumn{2}{|c|}{0} \\
\hline
\end{tabular}

Letras iguais maiúsculas na linha não diferem entre para teste de Tukey $(P \leq 0,05)$.

O controle de Bidens pilosa avaliado está descrito na Tabela 4. Não houve significância para os volumes, ou seja, a redução no volume de calda não interfere negativamente na eficiência do controle químico. Para os horários de aplicação houve melhor eficácia quando 
os produtos foram às $10 \mathrm{~h}$ nas avaliações de sete e 14 dias após a aplicação, aos 21 dias após a aplicação houve o controle satisfatório de $85 \%$ do picão preto. Tomazela et al. (2006) avaliou diferentes volumes de calda no controle de Brachiaria plantaginea, observou que a quantidade de calda depositadas por plantas e por $\mathrm{cm}^{2}$ de área foliar foram maiores quando se utilizaram maiores volumes de calda.

Tabela 4: Efeito do volume e horário de aplicação no controle da Bidens pilosa aos 7, 14 e 21 dias após aplicação de herbicida. Amambai-MS, 2017.

\begin{tabular}{|c|c|c|c|c|c|c|}
\hline \multirow{3}{*}{$\begin{array}{l}\text { Volume } \\
\left(\mathrm{Lha}^{-1)}\right.\end{array}$} & \multicolumn{6}{|c|}{ Controle $(\%)$} \\
\hline & \multicolumn{2}{|c|}{7 dias } & \multicolumn{2}{|c|}{14 dias } & \multicolumn{2}{|c|}{21 dias } \\
\hline & $7 \mathrm{~h}$ & $10 \mathrm{~h}$ & $7 \mathrm{~h}$ & $10 \mathrm{~h}$ & $7 \mathrm{~h}$ & $10 \mathrm{~h}$ \\
\hline 160 & $70,3 \mathrm{~B}$ & $73,3 \mathrm{~A}$ & $77,0 \mathrm{~B}$ & $78,0 \mathrm{~A}$ & $79,3 \mathrm{~B}$ & $85,3 \mathrm{~A}$ \\
\hline 130 & $71,3 \mathrm{~B}$ & $73,3 \mathrm{~A}$ & $76,3 \mathrm{~B}$ & $78,3 \mathrm{~A}$ & $79,3 \mathrm{~B}$ & $85,3 \mathrm{~A}$ \\
\hline 100 & $70,3 \mathrm{~B}$ & $73,3 \mathrm{~A}$ & $76,3 \mathrm{~B}$ & $78,6 \mathrm{~A}$ & $79,6 \mathrm{~B}$ & $85,6 \mathrm{~A}$ \\
\hline $\begin{array}{c}\text { CV horários } \\
(\%)\end{array}$ & \multicolumn{2}{|c|}{0,69} & \multicolumn{2}{|c|}{0,61} & \multicolumn{2}{|c|}{0,45} \\
\hline $\begin{array}{c}\text { CV volumes } \\
(\%)\end{array}$ & \multicolumn{2}{|c|}{0.80} & \multicolumn{2}{|c|}{1,01} & \multicolumn{2}{|c|}{1,11} \\
\hline
\end{tabular}

Letras iguais maiúsculas na linha não diferem entre para teste de Tukey $(P \leq 0,05)$.

O controle de Cenchrus echinatus avaliado está descrito na Tabela 5. Assim como nas outras espécies estudadas não houve diferença significativa para os volumes, o que infere que a redução no volume de calda não interfere negativamente na eficiência do controle químico. Para os horários de aplicação houve melhor eficácia quando os produtos foram às 10 horas nas avaliações de Sete e 14 dias após a aplicação, aos 21 dias após a aplicação houve o controle de $100 \%$ do capim-carrapicho.

A utilização de menor volume de calda é viável e reduz custos de transporte de água no campo e a perda de tempo representada pelas constantes paradas para reabastecimento do pulverizador, além de diminuir o risco de perdas por escorrimento (RODRIGUES, et al., 2011). De acordo com Silva et al. (2009), essa diminuição de perdas por escorrimento significaria, certamente, menor contaminação de águas superficiais.

Não foram observadas diferenças significativas entre os volumes de aplicação estudados, assim possibilitando o uso de 100,130 e $160 \mathrm{~L} \mathrm{ha}^{-1}$ de caldas aplicados nos horários mais favoráveis a cada um dos herbicidas. Observou-se que o decréscimo do volume de aplicação não é sinônimo de decréscimo de controle, as três plantas daninhas obtiveram um controle acima de $85 \%$ aos 21 dias após aplicação.

Os horários de aplicação tiveram diferenças para todas as plantas daninhas avaliadas, atingindo $100 \%$ de controle às 10h para Digitaria horizontalis e Cenchrus echinatus. Para Bidens pilosa o nível não chegou a $100 \%$ para nenhum dos horários observados, mas entre eles teve uma significativa diferença de controle, sendo para o horário das $10 \mathrm{~h}$ o melhor pois as condições climáticas têm papel decisivo na eficiência de aplicação. De acordo com Silveira (2001), o clima tem papel fundamental na efetividade de uma aplicação, em que de maneira geral temperaturas inferiores a $30{ }^{\circ} \mathrm{C}$, umidade superior a $55 \%$ e ventos de 2,0 a $3,2 \mathrm{~km} \mathrm{~h}^{-1}$ ou menores são condições favoráveis à pulverização com menores riscos de deriva e diminuição do tempo de vida da gota.

Tabela 5: Efeito do volume e horário de aplicação no controle da Cenchrus echinatus aos 7, 14 e 21 dias após aplicação de herbicida. Amambai-MS, 2017.

\begin{tabular}{|c|c|c|c|c|c|c|}
\hline \multirow{3}{*}{$\begin{array}{l}\text { Volume } \\
\left(\mathrm{Lha}^{-1)}\right.\end{array}$} & \multicolumn{6}{|c|}{ Controle $(\%)$} \\
\hline & \multicolumn{2}{|c|}{7 dias } & \multicolumn{2}{|c|}{14 dias } & \multicolumn{2}{|c|}{21 dias } \\
\hline & $7 \mathrm{~h}$ & $10 \mathrm{~h}$ & $7 \mathrm{~h}$ & $10 \mathrm{~h}$ & $7 \mathrm{~h}$ & $10 \mathrm{~h}$ \\
\hline 160 & $10,6 \mathrm{~B}$ & $15,6 \mathrm{~A}$ & $97,0 \mathrm{~B}$ & $99,3 \mathrm{~A}$ & $99,6 \mathrm{~B}$ & $100 \mathrm{~A}$ \\
\hline 130 & $10,6 \mathrm{~B}$ & $15,6 \mathrm{~A}$ & $97,3 \mathrm{~B}$ & $99,0 \mathrm{~A}$ & $100 \mathrm{~B}$ & $100 \mathrm{~A}$ \\
\hline 100 & $10,3 \mathrm{~B}$ & $15,6 \mathrm{~A}$ & $97,3 \mathrm{~B}$ & $99,0 \mathrm{~A}$ & $99,6 \mathrm{~B}$ & $100 \mathrm{~A}$ \\
\hline $\begin{array}{c}\text { CV horários } \\
(\%)\end{array}$ & \multicolumn{2}{|c|}{6,1} & \multicolumn{2}{|c|}{0,51} & \multicolumn{2}{|c|}{0,37} \\
\hline $\begin{array}{c}\text { CV volumes } \\
(\%)\end{array}$ & \multicolumn{2}{|c|}{3,6} & \multicolumn{2}{|c|}{0,42} & \multicolumn{2}{|c|}{0,33} \\
\hline
\end{tabular}

Letras iguais maiúsculas na linha não diferem entre para teste de Tukey $(P \leq 0,05)$.

\section{Considerações finais}

A qualidade na aplicação expressa em DMV, volume recuperado, porcentagem de área coberta e densidade de gotas foi melhor na aplicação realizada às 10 horas. $\mathrm{O}$ controle de plantas daninhas foi eficiente independente do volume de aplicação, e foi mais eficaz no horário das 10 horas para Cenchrus echinatus, Bidens pilosa e Digitaria horizontalis.

\section{Referências}

ADEGAS, F. S. Pontos relevantes na tecnologia de aplicação de agrotóxicos. Embrapa soja, Londrina, 23 de novembro de 2016. Disponível em:<blogs.canalrural.com.br/embrapasoja/2016/11/23/ pontos-relevantes-na-tecnologia-de-aplicacao-deagrotoxicos/>. Acesso em: 28 de fevereiro de 2020.

ALMEIDA, D.P.; TIMOSSI, P. C.; LIMA, S. F.; SILVA, U. R. REIS, E. F. Condições atmosféricas e volumes de aplicações na dessecação de Urochloa 
ruziziensis e vegetação espontânea. Revista Brasileira de Herbicidas, v.13, n.3, p.245-251, 2014.

ANTANIASSI, U. R. Qualidade em tecnologia de aplicação de defensivos. Anais do $\mathbf{V}$ Congresso Brasileiro de Algodão, FCA/ UNESP, BOTUCATU/SP, 2015.

ANTUNIASSI, U.R.; BAIO, F.H.R. Tecnologia de aplicação de agrotóxicos. In: VARGAS, L.; ROMAN, E.S. (Org.). Manual de manejo e controle de plantas daninhas. Passo Fundo: Embrapa, 2008, p.173-212.

CHRISTOFFOLETI, P. J. (Coord.). Aspectos de resistência de plantas daninhas a herbicidas. Campinas: Associação Brasileira de Ação a Resistência de Plantas aos Herbicidas (HRAC-BR), 2004, p. 3-22.

CUNHA, J.P.A.R. Simulação de deriva de agrotóxicos em diferentes condições de pulverização. Ciência e agrotecnologia, v. 32, n. 5, p. 1616-1621, 2008.

DEVLIN, D.L. Using reduced rates of postemergence herbicides in soybeans (Glycine max). Weed Technology, v.5, p. 834-840, 1991.

EMBRAPA - Empresa Brasileira de Pesquisa Agropecuária. Gotas: sistema de análise de deposição de agrotóxicos. Informática Agropecuária, Jaguariúna, SP, 2006.

FAVARETTO, L.A.Influência do horário de aplicação de fungicidas sobre o progresso de ferrugem asiática e produtividade de soja. 2019.23f.Monografia (Bacharelado em Agronomia). Universidade Federal da Fronteira Sul, Erechim.

FOLONI, L. L. Impacto ambiental do uso de herbicidas. In: CONGRESSO BRASILEIRO DA CIÊNCIA DAS PLANTAS DANINHAS, 22., 2000, Foz-do-Iguaçu. Londrina. Anais... Sociedade Brasileira da Ciência das Plantas Daninhas, 2000, p. 49-91.

FRIEDRICH, T. Quality in pesticide application technology. In: RAETANO, C.G.; ANTUNIASSI, U. R. Qualidade em tecnologia de aplicação. Botucatu: Fepaf, 2004, p. 93-109.

IBGE. Instituto Brasileiro de Geografia e Estatística. Agência IBGE Notícias. Em Julho, IBGE prevê safra de grãos 31,1\% maior que em 2016. Disponível em: <https://www.noticiasagricolas.com.br/noticias/agrone gocio/196831-em-julho-ibge-preve-safra-de-graos311-maior-que-em-2016.html\#.WgYKOm6nHIU> Acesso em: nov. 2017.
LORENZI, H. Manual de identificação e controle de plantas daninhas: plantio direto e convencional. Nova Odessa, SP: Instituto Plantarum, 2014, p.384.

MATTHEWS, G. A. et al. Métodos de aplicação de defensivos agrícolas. São Paulo: Andrei, 2016.

MATTHEWS, G. A. et al. Pesticide application methods, Blackwell: 2014.

MATUO, T. Técnicas de aplicação de defensivos agrícolas. Jaboticabal: FUNEP, 1990.

NASCIMENTO, J. M.; GAVASSONI, W. L.; SOUZA, C. M. A.; BACCHI, L. M. A.; SERRA, A. P.; ZACCARON, M. L. Pontas de pulverização e horários de aplicação no controle químico de ferrugem asiática da soja. Semina: Ciência Agrária, Londrina, v.34, n. 5, p. 2037-2048, 2013.

NASCIMENTO, J. M.; SOUZA, C.M.A.; GAVASSONI, W. L.; BACCHI, L. M. A.; SERRA, A. P.; FENGLER, G.W. Controle de ferrugem asiática da soja utilizando-se diferentes pontas de pulverização em Maracaju-MS. Revista Ciências Técnicas Agropecuária, v.18, n.1, p.1-6, 2009.

NIEWEGLOWSKI FILHO, M.; PELISSARI, A.; KOEHLER, H. S.; BASSETTI, J.C.; MURARO, M.; KERKHOFF, M.; SPHYRA, A. Controle químico de plantas daninhas utilizando diferentes pontas de pulverização. Scientia Agraria, v.15, n.1, p.33-37, 2014.

PENCKOWSKI, L. H.; PODOLAN, M. J.; LÓPEZOVEJERO, R. F. Influência das condições climáticas no momento da aplicação de herbicidas pósemergentes sobre a eficácia de controle de nabiça (Raphanus raphanistrum) na cultura do trigo. Planta Daninha, v. 21, n. 3, p. 435-442, 2003.

QASEM, J. R. Herbicides Applications: Problems and Considerations, Herbicides and Environment. InTech, v.4, n.32, p.643-665. 2011.

RAMIRES, A.C.; CONSTANTIN, J.; MARCHIORI JUNIOR, O.; MACIEL, C.D. de G.; OLIVEIRA JUNIOR, R. S. de O.; APOLONI, D.K.M. Influência dos diferentes horários de aplicação em pós-emergência dos herbicidas chlorimuron-ethyl, fomesafen e bentazon no controle Commelina benghalensis L. Acta Scientiarum, v. 21, n.3, p.467-472, 1999.

RODRIGUES, E. B.; SAAB, O. J. G.; GANDOLFO, M. A. Cana-de-açúcar: avaliação da taxa de aplicação e 
deposição do herbicida glyphosate. Revista Brasileira de Engenharia Agrícola e Ambiental, v. 15, n. 1, p. 90-95, 2011.

SANTOS, J. M. F. dos. Mini curso: tecnologia de aplicação de pesticidas (terrestre e aérea). Tecnologia de Aplicação de Defensivos Agrícolas. São Paulo, SP, 2005.

SCHAMPHELEIRE, M. de.; NUYTTENS, D.; BAETENS, K.; CORNELIS, W. M.; GABRIELS, D.; SPANOGUE, P. Effects on pesticide spray drift of the physicochemical properties of the spray liquid. Precision agricultural, v. 10, n. 5, p. 409-420, 2009.

SILVA, A. A. da; SILVA, J. F.; FERREIRA, F. A.; FERREIRA, L. R.; SILVA, J. F.; Colaboradores: OLIVEIRA JÚNIOR, R. S. de; VARGAS, L. Controle de plantas daninhas. Brasília: ABEAS; Viçosa, MG: DFTIUFV, $1999.260 \mathrm{p}$.

SILVA, A. A. et al. Tópicos em manejo de plantas daninhas. Viçosa, MG: EFV, 2009.

TOMAZELA, M.S.; MARTINS, D.; MARCHI, S.R.; NEGRISOLI, E. Avaliação da deposição da calda de pulverização em função da densidade populacional de brachiaria plantaginea, do volume e do ângulo de aplicação. Planta Daninha, v. 24, n. 1, p. 183-189, 2006.

VELINI, E. D. et al. Procedimentos para instalação, avaliação e análise de experimentos com herbicidas. Londrina: SBCP, 1995. 